\title{
Fuzzy Multiobjective Reliability Optimization Problem of Industrial Systems Using Particle Swarm Optimization
}

\author{
Harish Garg \\ Department of Mathematics, Indian Institute of Technology Roorkee, Roorkee, Uttarakhand 247667, India \\ Correspondence should be addressed to Harish Garg; harishg58iitr@gmail.com
}

Received 9 February 2013; Accepted 15 April 2013

Academic Editor: Tamer Eren

Copyright (C) 2013 Harish Garg. This is an open access article distributed under the Creative Commons Attribution License, which permits unrestricted use, distribution, and reproduction in any medium, provided the original work is properly cited.

\begin{abstract}
The present work investigates the reliability optimization problem of the repairable industrial systems by utilizing uncertain, limited, and imprecise data. In many practical situations where reliability enhancement is involved, the decision making is complicated because of the presence of several mutually conflicting objectives. Moreover, data collected or available for the systems are vague, ambiguous, qualitative, and imprecise in nature due to various practical constraints and hence create some difficulties in optimizing the design problems. To handle these problems, this work presents an interactive method for solving the fuzzy multiobjective optimization decision-making problem, which can be used for the optimization decision making of the reliability with two or more objectives. Based on the preference of the decision makers toward the objectives, fuzzy multi-objective optimization problem is converted into crisp optimization problem and then solved with evolutionary algorithm. The proposed approach has been applied to the decomposition unit of a urea fertilizer plant situated in the northern part of India producing 1500-2000 metric tons per day.
\end{abstract}

\section{Introduction}

Reliability in general can be defined as the ability of a system to perform its required functions under stated conditions for a specified period of time. Reliability technology is an important phenomenon and is widely used for increasing the efficiency, risk analysis, production availability studies, and design of industrial systems. The industrial systems run continuously and suffer failure over a period of time which can be brought back in to service by proper repair or replacement. Consequently, it may be extremely difficult, if it is not possible to construct accurate and complete mathematical model for the system in order to access the reliability because of inadequate knowledge about the basic failure events [1, 2]. In highly competitive industrial market, the concept of failure analysis is an unavoidable fact in complex industrial systems. Reliability of such systems not only depends on the reliability of each element of these systems but also depends on occurrence of sequence of failures.

Generally, the design problems are always stated in precise mathematical forms. It must be recognized that many practical problems encountered by designers and decision makers would take place in an environment in which the statements might be vague or imprecise. Usually, it is difficult to describe the goals and constraints of such optimization problems by crisp relations through equations and/or descriptions. In such situations, the traditional reliability theory, based on probabilistic and binary state assumptions, does not always provide useful information to the practitioners due to the limitation of being able to handle only quantitative information [3-5]. An alternative for this fuzzy set theory [6] can build a model to represent a subjective estimation of possible effect of the given values on the problem and permit the incorporation of vagueness in the conventional set theory that can be used to deal with uncertainty quantitatively. Due to incomplete and uncertain input information, mathematical models of such problems are developed in fuzzy environment, and the optimization problem under consideration becomes a fuzzy programming problem. The fuzzy set-based optimization was firstly introduced by Bellman and Zadeh [7] in their seminal paper on decision making in a fuzzy environment, in which the concepts of fuzzy constraint, 
fuzzy objective, and fuzzy decision were introduced. After this pioneering work, these concepts were subsequently profusely used and applied by many investigators. In most of the practical design situation, the presence of several conflicting objectives which are nonlinear and ambiguous complicates the reliability apportionment. For instance, a designer is required to minimize the system cost while simultaneously maximizing the system reliability. Therefore, multiobjective functions become an important aspect in the reliability design of the engineering systems, and hence various researchers [8-15] used different methods to solve reliability optimization problem in fuzzy environment.

In multiobjective optimization problems (MOOPs), it is difficult or rarely possible to find an optimal solution for all the objectives which simultaneously optimize the problem in fuzzy environment. For handling such types of situations, one usually tries to search for a solution which is as close to the decision makers (DMs) expectations as possible. For this reason, problem is solved is an interactive manner in which DM is initially asked to specify his or her preferences towards the objectives. Based on these preferences, the problem is solved, and the DM is provided with a possible solution. If the DM is satisfied with this solution, the problem ends there; otherwise, he or she is asked to modify his or her preferences in the light of the earlier obtained results. This iterative procedure is continued till a satisfactory solution is achieved which is close to DM's expectations.

The present work is an extension of the work earlier done by Sharma and Garg [16] and Kumar [17], in which the cost factor was not considered in mathematical modeling. In this study, a conflicting multiobjective nonlinear programming problem is considered in fuzzy environment where we maximize the reliability and minimize the cost of the system. A conflicting nature between the objectives is resolved with the help of the fuzzy technique. Also, the intention is to use compensatory operator for aggregation of the different fuzzy goals and a robust global optimization technique, namely particle swarm optimization (PSO), for solution of the resultant single objective optimization problem thus formulated with the choice of the DM/system expert regarding the priority amongst the objectives. The technique is explained through a case study of decomposition unit of a fertilizer plant, a complex repairable industrial system. The rest of the chapter is organized as follows. Section 2 describes the general definitions related to multiobjective optimization problem, while corresponding reliability optimization model is given in Section 3. The solution procedure for solving the multiobjective reliability optimization problem is described in Section 4. A system description for illustrating the approach has been given in Section 5, while their corresponding results and analysis are done in Section 6. Finally, some concrete conclusions are drawn and are presented in Section 7.

\section{A General Multiobjective Nonlinear Programming Problem}

A multiobjective nonlinear programming problem is to find the solution vector or vector of design variables $x=\left(x_{1}, x_{2}, \ldots, x_{K}\right)^{T}$ that optimizes a vector of objective functions

$$
f(x)=\left\{f_{1}(x), f_{2}(x), \ldots f_{b}(x)\right\}
$$

over the feasible design space $X$. The problem is modeled as follows (WLOG we assume the minimization):

$$
\begin{array}{ll}
\text { Minimize: } & f(x) \\
\text { subject to: } & h_{v}(x)=0, \quad v=1,2, \ldots, I \\
& g_{j}(x) \leq 0, \quad j=1,2, \ldots, J \\
& x_{k}^{l} \leq x_{k} \leq x_{k}^{u}, \quad k=1,2, \ldots, K,
\end{array}
$$

where $f_{1}(x), f_{2}(x), \ldots, f_{b}(x)$ are the individual objective functions; $h_{\nu}(x)$ and $g_{j}(x)$ are equality and inequality constrained functions, respectively. $x_{k}^{l}$ and $x_{k}^{u}$ are the lower and upper bounds of decision variable $x_{k}$, respectively. The concept of optimality in single objective is not directly applicable in MOOPs. For this reason, a classification of the solutions is introduced in terms of Pareto optimality according to the following definitions, in terms of minimization.

Definition 1 (Pareto optimal). A solution vector $x^{*} \in X$ is Pareto optimal solution if there does not exist another point $x \in X$ such that $f_{t}(x) \leq f_{t}\left(x^{*}\right)$ for all $t=1,2, \ldots, b$ and $f_{s}(x)<f_{s}\left(x^{*}\right)$ for at least one $s$. Such solutions are also called true Pareto optimal solution.

Definition 2 (Pareto dominance). A dominance $x$ dominates $y$ denoted as $x>y$ if and only if $f_{t}(x) \leq f_{t}(y)$, and there exists $q$ s.t. $f_{q}(x)<f_{q}(y), t, q \in\{1,2, \ldots, b\}$. If there are no solutions which dominate $x$, then $x$ is nondominated.

Definition 3 (Pareto set). A set of nondominated solutions $\left\{x^{*} \mid \neg \exists x: x>x^{*}\right\}$ are said to be a Pareto set.

Definition 4 (Pareto front). The set of vectors in the objective space that are an image of a Pareto set that is $\left\{f\left(x^{*}\right) \mid \neg \exists x\right.$ : $\left.x>x^{*}\right\}$.

In general, there exist a number of Pareto optimal solutions to a multiobjective optimization problems. Due to multiobjectives, the selection of such objectives clearly depends on the problem under study and the decision maker (DM) criteria. Thus, the designer must select a compromise or a satisfying solution from the Pareto optimal solution set according to his or her preference.

\section{Reliability Optimization Model}

Reliability is one of the vital attributes of performance in arriving at the optimal design of a system because it directly and significantly influences the system's performance. In practical, the problem of system reliability may be formed as a typical nonlinear programming problem with nonlinear costfunctions in fuzzy environment. 
3.1. Formulation of System Reliability Model. Let us consider that the reliability problem of a system consists of $n$ components. Each component has reliability $R_{i}$ for the $i$ th components for $i=1,2, \ldots, n$. Then, the system reliability is written in the form of reliability of each component as

$$
\begin{aligned}
& R_{s}\left(R_{1}, R_{2}, \ldots, R_{n}\right) \\
& \quad= \begin{cases}\prod_{i=1}^{n} R_{i} & \text { for series system } \\
1-\prod_{i=1}^{n}\left(1-R_{i}\right) & \text { for parallel system } \\
\text { or combination of series } & \\
\text { and parallel system. } & \end{cases}
\end{aligned}
$$

According to Aggarwal and Gupta [18], the cost of reliability is monotonically increasing function of reliability and hence based on the fact that the $i$ th components reliability cost is $C_{i}\left(R_{i}\right)$. Therefore, the system cost is given by

$$
C_{s}\left(R_{1}, R_{2}, \ldots, R_{n}\right)=\sum_{i=1}^{n} C_{i}\left(R_{i}\right) .
$$

In reliability optimization problems, it is often required to minimize or maximize several objectives subject to several constraints. For instance, a designer is required to minimize the system cost while simultaneously maximizing the system reliability. Therefore, multiobjective functions become an important aspect in the reliability design of the engineering systems. Hence, the suitable form of optimization model of series system reliability problem by considering the system reliability and cost as objective is

Maximize:

$$
\begin{aligned}
R_{s}\left(R_{1}, R_{2}, \ldots, R_{n}\right) & \begin{cases}\prod_{i=1}^{n} R_{i} & \text { for series system } \\
1-\prod_{i=1}^{n}\left(1-R_{i}\right) & \text { for parallel system } \\
\text { or combination of series } & \\
\text { and parallel system } & \end{cases}
\end{aligned}
$$

Minimize: $\quad C_{s}\left(R_{1}, R_{2}, \ldots, R_{n}\right)=\sum_{i=1}^{n} C_{i}\left(R_{i}\right)$

subject to $\quad R_{i, \min } \leqslant R_{i} \leqslant 1, R_{s, \min } \leqslant R_{s} \leqslant 1$

$$
\text { for } i=1,2, \ldots, n \text {. }
$$

\subsection{System Reliability Optimization Model in Fuzzy Environ-} ment. The necessary features of the cost versus maintainability function are equivalent to the cost versus reliability function as given by Aggarwal and Gupta [18]. It is very complicated decision-making process to determine the reliability components in fuzzy objective as well as constraint goal. It involves many uncertain factors and becomes a nonstochastic vague decision-making process. Therefore, the reliability allocation model (5) can be represented by fuzzy nonlinear programming to make the model more flexible and adoptable to the human decision process. Therefore, in fuzzy environment, the optimization problem (5) becomes

$$
\begin{aligned}
& \text { Max: } \quad\left\{R_{s},-C_{s}\right\} \\
& \text { subject to } \quad R_{i, \min } \leqslant R_{i} \leqslant 1, \quad R_{s, \text { min }} \leqslant R_{s} \leqslant 1 \\
& \\
& \text { for } i=1,2, \ldots, n .
\end{aligned}
$$

The symbol $\widetilde{\operatorname{Max}}$ denotes a relaxed or fuzzy version of "Max."

\section{Interactive Methods for Solving Multiobjective Reliability Optimization Problems}

Multiobjective optimization is intensively used in engineering applications for simultaneously optimizing the collection of objective functions systematically. In general, reliability optimization problem is solved with the assumption that the coefficients or cost of components is specified in a precise way. In real life, there are many diverse situations due to uncertainty in judgments, lack of evidence, and so forth such that there are incompleteness and unreliability of input information, and hence it is not possible to get relevant precise data for the reliability system. These types of impreciseness in data are well handled with the help of defining their membership functions by fuzzy set theory instead of representing by random variable. Thus, due to these, the concerned optimization problems are modeled in fuzzy environment, and hence the problem under consideration becomes a fuzzy programming problem. But in a multiobjective optimization problem, an optimal solution which simultaneously optimizes all the objectives, and that too when the problem is modeled in a fuzzy environment, is rarely possible. In such situations, one usually tries to search for the best possible solution in the presence of imprecise information which is as close to the DM's expectations as possible. Search of such a satisfying solution requires solving the multiobjective fuzzy optimization problem iteratively in an interactive manner, wherein the DM is initially asked to specify his or her preferences and expectations. Based on these preferences, the problem is solved, and the DM is provided with a possible solution. If the DM is satisfied with this solution, the problem ends there; otherwise, he or she is asked to modify his or her preferences in the light of the earlier obtained results. This iterative procedure is continued till a satisfactory solution is achieved which is close to DM's expectations. The detail of the computational procedure for solving the fuzzy multiobjective reliability optimization problem in an iterative way is described in the following steps.

Step 1 (find the ideal and anti-ideal values of each objective function). First step in the proposed technique is to find the minimal and maximal feasible values of the objective functions. For this reason each objective function has been solved separately by taking single objective optimization 
problem under given set of constraints. The corresponding solutions thus obtained are known as ideal solutions. Based on these solutions, $\left(R_{1}^{*}\right.$ and $\left.R_{2}^{*}\right)$, minimal and maximal values of each objective are calculated as

$$
\begin{aligned}
& R_{s}^{l}=\min \left\{R_{s}\left(R_{1}^{*}\right), R_{s}\left(R_{2}^{*}\right)\right\}, \\
& R_{s}^{u}=\max \left\{R_{s}\left(R_{1}^{*}\right), R_{s}\left(R_{2}^{*}\right)\right\}, \\
& C_{s}^{l}=\min \left\{C_{s}\left(R_{1}^{*}\right), C_{s}\left(R_{2}^{*}\right)\right\}, \\
& C_{s}^{u}=\max \left\{C_{s}\left(R_{1}^{*}\right), C_{s}\left(R_{2}^{*}\right)\right\},
\end{aligned}
$$

where $R_{1}^{*}$ and $R_{2}^{*}$ are the ideal solutions of the objective functions corresponding to minimization and maximization, respectively.

Step 2 (establishing the fuzzy goals towards the objective functions). In the traditional optimization, the design feasibility is considered as either satisfied or violated. For many engineering applications, the transition from infeasibility to feasibility is not obvious, because of not only the vague information in the design constraints but also the factors that can affect the design scenario, such as designer's knowledge, manufacture precision, and material properties. For this reason, they are modeled in such a way that the transition from infeasible state to feasible state is smooth and gradual with subjectivity. To incorporate the DM's vague idea about in which region of the objective the optimum should be, the degree of fuzziness is used. Essentially, the approach is to translate the functions to a common scale $[0,1]$ by the means of mathematical transformations, combine them using the geometric mean, and optimize the overall metric. In this paper, the problem is fuzzified with the help of linear membership functions. Let $\widetilde{R}_{s}$ and $\widetilde{C}_{s}$ be the fuzzy region of satisfaction of system reliability $\left(R_{s}\right)$ and system cost $\left(C_{s}\right)$, respectively, and let $\mu_{R_{s}}$ and $\mu_{C_{s}}$ be their corresponding membership functions. Then, the fuzzy objective stated by a designer can be quantified by eliciting corresponding linear membership functions, using the minimal and maximal feasible values of each objective as obtained during Step 1, and is defined as.

For maximization goal $\left(R_{s}\right)$

$$
\mu_{R_{s}}(x)= \begin{cases}1, & R_{s}(x) \geqslant R_{s}^{u} \\ \frac{R_{s}(x)-R_{s}^{l}}{R_{s}^{u}-R_{s}^{l}}, & R_{s}^{l} \leqslant R_{s}(x) \leqslant R_{s}^{u} \\ 0, & R_{s}(x) \leqslant R_{s}^{l} .\end{cases}
$$

Here, $\mu_{R_{s}}(x)$ is strictly monotonically increasing function of $R_{s}(x)$.

For minimization goal $\left(C_{s}\right)$

$$
\mu_{C_{s}}(x)= \begin{cases}1, & C_{s}(x) \leqslant C_{s}^{l} \\ \frac{C_{s}^{u}-C_{s}(x)}{C_{s}^{u}-C_{s}^{l}}, & C_{s}^{l} \leqslant C_{s}(x) \leqslant C_{s}^{u} \\ 0, & C_{s}(x) \geqslant C_{s}^{u} .\end{cases}
$$

Here, $\mu_{C_{s}}(x)$ is strictly monotonically decreasing function of $C_{s}(x)$.
Step 3 (equivalent single optimization problem). Using the achieved objectives' membership functions of $R_{s}$ and $C_{s}$, it is very important to choose the aggregation operator. Even since Zadeh [6] suggested that $\wedge$, that is, min be used for the intersection of fuzzy sets as

$$
\mu_{D}=\mu_{R_{s}} \wedge \mu_{C_{s}}
$$

The two objectives $\mu_{R_{s}}$ and $\mu_{C_{s}}$ in (10) are equally important. This is not true in a real-life situation; that is, DMs sometimes do not pay equal attention to these two objectives. Owing to this (10), is modified, according to the importance of the objective, by Huang [11] as

$$
\mu_{D}=\left(1 \wedge \frac{\mu_{R_{s}}}{w_{1}}\right) \wedge\left(1 \wedge \frac{\mu_{C_{s}}}{w_{2}}\right),
$$

where $w_{1}$ and $w_{2} \in[0,1]$ are called the objective weights. Thus, using the achieved objectives' membership functions and $\mathrm{DM} /$ system expert preferences in the form of weights $W=\left[w_{1}, w_{2}\right]$, problem is formulated as a single objective optimization problem and is given as

$$
\begin{array}{ll}
\text { Maximize: } & \mu_{D}(x)=\left(1 \wedge \frac{\mu_{R_{s}}(x)}{w_{1}}\right) \wedge\left(1 \wedge \frac{\mu_{C_{s}}(x)}{w_{2}}\right), \\
\text { Subject to } & x_{k}^{l} \leq x_{k} \leq x_{k}^{u}, \quad k=1,2, \ldots, K, \\
& w_{t} \in[0,1], \quad t=1,2,
\end{array}
$$

where $\wedge$ indicates the intersection or min operator, $w_{t}$ represents the $t$ th objective weight, in the form of the importance of the objective functions, suggested by DM, $x$ is the vector of decision variables, and $x_{k}^{l}$ and $x_{k}^{u}$ are the lower and upper bounds of decision vector $x_{k}$, respectively. The obtained optimization problem is solved with the particle swarm optimization algorithm which has been described in Section 4.1.

Step 4 (adjusting the preference parameters). If the DM is satisfied by the solution obtained in Step 3, then the approach stops successfully. Otherwise, the key preference parameters, that is, decision maker's preferences regarding the relative importance of each objective function $\left(W=\left[w_{1}, w_{2}\right]\right)$, can be altered to meet the DM's choice, and the method again goes back to Step 3. The process is repeated until DM is satisfied. We are just showing one run of the approach here as we assume that in this problem DM is satisfied by the results obtained in Step 3.

4.1. A Survey of PSO Algorithm. The particle swarm optimization (PSO) algorithm was firstly proposed by Kennedy and Eberhart $[19,20]$ and has deserved some attention during the last years in the global optimization field. PSO algorithm is based on the population of agents or particles and tries to simulate its social behavior in optimal exploration of problem space. The PSO algorithm is inspired by social behavior of bird flocking, animal hording, or fish schooling. In PSO, the potential solutions, called particles, fly through the problem 
space by following the current optimum particles. PSO is initialized with a group of random particles (solutions) and then searches for optima by updating generations. During every iteration, each particle is updated by following two "best" values. The first one is the position vector of the best solution (fitness), this particle has achieved so far. The fitness value is also stored. This position is called pbest. Another "best" position that is tracked by the particle swarm optimizer is the best position, obtained so far by any particle in the population. This best position is the current global best and is called gbest. After finding the two best values, the particle updates its velocity and position according to (13) and (14), respectively,

$$
\begin{gathered}
v_{k+1}^{i}=w * v_{k}^{i}+c_{1} * \mathrm{ud} *\left(\text { pbest }^{i}-x_{k}^{i}\right) \\
+c_{2} * \mathrm{Ud} *\left(\text { gbest }_{k}-x_{k}^{i}\right), \\
x_{k+1}^{i}=x_{k}^{i}+v_{k+1}^{i},
\end{gathered}
$$

where $v_{k}^{i}$ is the velocity of $i$ th particle at the $k$ th iteration and $x_{k}^{i}$ is currently the solution (or position) of the $i$ th particle. ud and Ud are random numbers generated uniformly between 0 and $1 . c_{1}$ is the self-confidence (cognitive) factor, and $c_{2}$ is the swarm confidence (social) factor. Finally, $w$ is the inertia factor that takes linearly decreasing values downward according to a predefined number of iterations. The first term in (13) represents the effect of the inertia of the particle, the second term represents the particle memory influence, and the third term represents the swarm (society) influence. The algorithm for the PSO can be summarized as follows.

(1) Initialize the swarm $x^{i}$, the position of particles is randomly initialized within the hypercube of feasible space.

(2) Set iteration counter $k=1$.

(3) Evaluate the performance $f$ of each particle, using its current position $x^{i}$.

(4) Compare the performance of each individual to its best performance so far. If $f\left(x^{i}\right)<f\left(\right.$ pbest $\left.t^{i}\right)$, then $f\left(\right.$ pbest $\left.^{i}\right)=f\left(x^{i}\right)$, pbest $^{i}=x^{i}$.

(5) Compare the performance of each particle to the global best particle. If $f\left(x^{i}\right)<f$ (gbest), then $f($ gbest $)=f\left(x^{i}\right)$, gbest $=x^{i}$.

(6) Update the velocity of the particle according to (13).

(7) Move each particle to a new position using (14).

(8) Update iteration counter by 1 ; that is, $k=k+1$.

(9) Go to Step 3, and repeat until convergence.

\section{Illustrative Example}

To illustrate, a fertilizer plant situated in the northern part of India and producing approximately 1500-2000 metric tons per day has been considered as a main system [17]. The brief description of the system is given below.
5.1. System Description. The fertilizer plants are large, complex, and repairable engineering unit which is a combination of ammonia and urea plant. The urea plant is composed of synthesis, decomposition, crystallization, and prilling system, arranged in predetermined configuration [16, 17]. In this process, the ammonia and $\mathrm{CO}_{2}$ enter the urea synthesis reactor. The reactants from urea synthesis reactor enter the urea decomposer in which urea is separated from reactants. These are further sent to urea crystallizer in which the urea solution is concentrated and crystallized. The urea crystals are separated by centrifuge and conveyed pneumatically to the top of urea prilling system. In this system, urea crystals are melted and sprayed through distributers and fall down in urea prilling tower against the ascending air allowing getting prilled on the way. The prilled urea is collected at the bottom of urea prilling, system and sent to bagging section. Among the various functional units in the plant such as urea synthesis, urea decomposition, urea crystallization, urea prilling and urea recovery, urea decomposition is one of the most important and vital functional processes which is the subject of our discussion.

5.2. Decomposition System. The gas-liquid mixture (urea, $\mathrm{NH}_{3}, \mathrm{CO}_{2}$, Biuret) flows from a reactor at $126^{\circ} \mathrm{C}$ into the upper part of a high-pressure decomposer, where the flashed gases are separated. The liquid falls through a sieve plate, which comes into contact with high-temperature gas available from the reboiler and the falling film heater. The same process is repeated in a low-pressure absorber. In the reboiler, the liquid is further heated to $151^{\circ} \mathrm{C}$ with medium-pressure steam, so that the remaining ammonia and carbonate are released as gases. The solution is then further heated to $165^{\circ} \mathrm{C}$ in the falling film heater, which reduces the Biuret formation and hydrolysis of urea. The overhead gases from the highpressure decomposer go to the high-pressure absorber cooler. The liquid flows to the top of the low-pressure absorber and is cooled in a heat exchanger. Additional flashing of the solution takes place in the upper part of the low-pressure absorber to reduce the solution pressure from 17.5 to $2.5 \mathrm{~kg} / \mathrm{cm}^{2}$. The low-pressure absorber has four sieve trays and a packed bed. In the packed bed, the remaining ammonia is stripped off by $\mathrm{CO}_{2}$ gas. The overhead gases go to the low-pressure absorber cooler, in which the pressure is controlled at $2.2 \mathrm{~kg} / \mathrm{cm}^{2}$. Most of the excess ammonia and carbonate are separated from the solution flowing to the gas separator. The gas separator has two parts: (a) the upper part is at $105^{\circ} \mathrm{C}$ and $0.3 \mathrm{~kg} / \mathrm{cm}^{2}$; here the remaining small amounts of ammonia and $\mathrm{CO}_{2}$ are recovered by reducing the pressure; the sensible heat of solution is enough to vaporize these gases; (b) the lower part has a packed section at $110^{\circ} \mathrm{C}$ and atmospheric pressure. Air containing a small amount of ammonia and $\mathrm{CO}_{2}$ is fed off from the gas absorber by an off-gas blower to remove the remaining small amounts of ammonia and $\mathrm{CO}_{2}$ present on the solution. Off-gases from the lower and upper parts are mixed and fed to the off-gas condenser. The urea solution, concentrated to $70-75 \%$, is fed to a crystallizer. In brief, the various subsystems and the components associated with them are defined as below. 
TABLE 1: Input data for decomposition system.

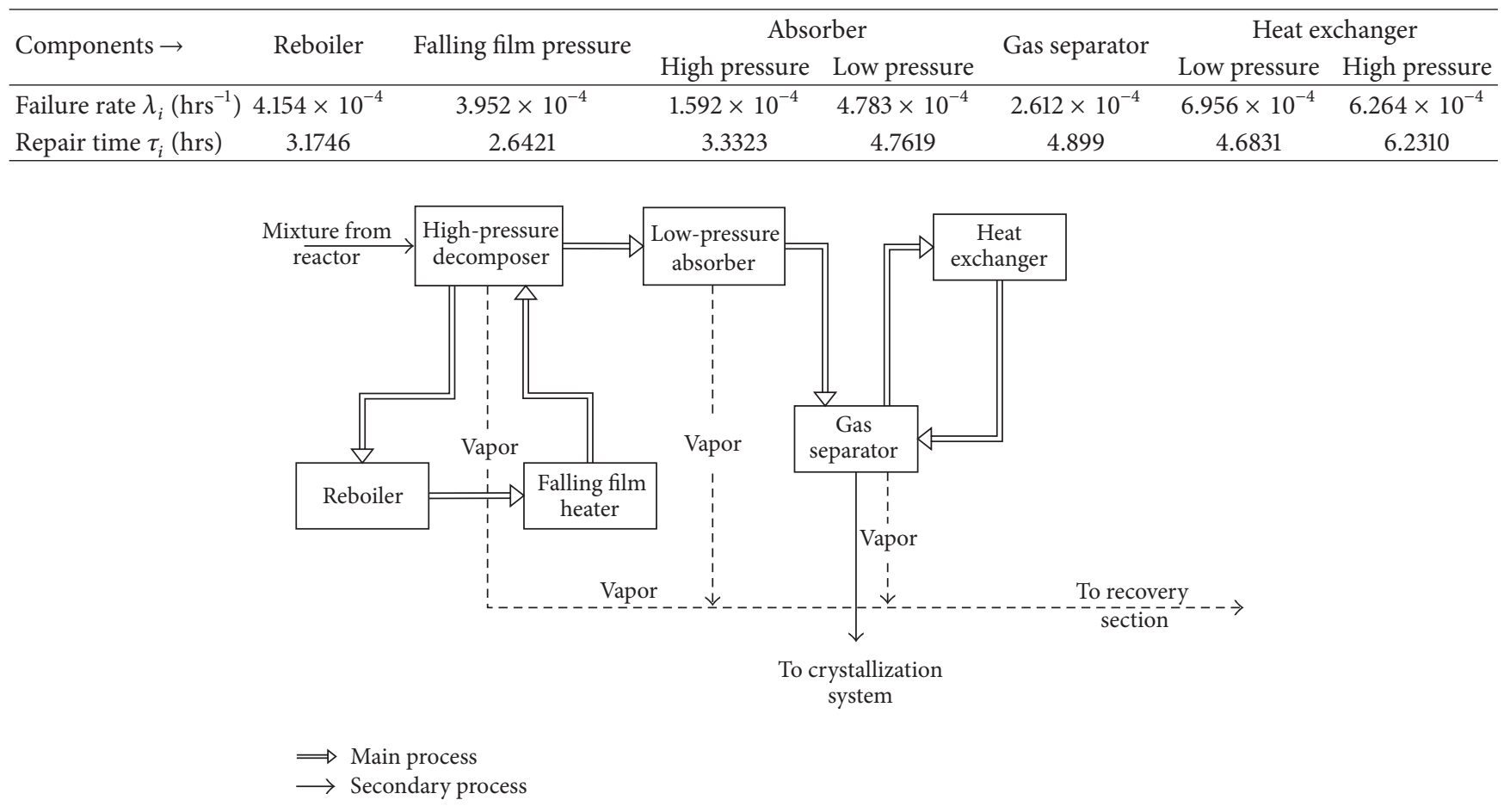

FIgURE 1: Schematic diagram of the decomposition unit.

(i) Subsystem $A_{i}$ has two units. Unit $A_{1}$ is called the reboiler for the high-pressure absorber, and unit $A_{2}$ is called the falling film heater for the low-pressure absorber. Failure of $A_{1}$ or $A_{2}$ causes complete failure of the system.

(ii) Subsystem $B_{i}$ has two units in series. Unit $B_{1}$ is called high-pressure absorber, and $B_{2}$ is called lowpressure absorber (the component contained of sieve trays and packed bed for tripping off the remaining ammonia). Failure of either unit causes the failure of whole system.

(iii) Subsystem $C$, the gas separator, has one unit only (used to separate the gases obtained from pressure absorbers. The solution is fed to crystallization unit for further processing) arranged in series with $B_{1}$ and $B_{2}$.

(iv) Subsystem $E_{i}$ has two units in series that is $E_{1}$ and $E_{2}$ where $E_{1}$ is low-pressure heat exchanger, and $E_{2}$ is high-pressure heat exchanger with standby unit (the heat exchangers are used to recover the heat of the gases). Failure of both at a time will cause complete failure of the system.

The schematic diagram of the system is shown in Figure 1. The data related to main components of the system, in the form of failure rate $\left(\lambda_{i}\right.$ 's) and repair time $\left(\tau_{i}\right.$ 's $\left.s\right)$, is collected from the historical or available records of the industry and is integrated with expertise of maintenance personnel which is given in Table 1 [16].
5.3. Reliability Optimization Problem. The multiobjective reliability optimization problem for the considered system is formulated by taking systems reliability and cost as an objective and crisp failure rates $\left(\lambda_{i}\right.$ 's $)$ and repair times $\left(\tau_{i}\right.$ 's) as decision variables under the considered $\pm 15 \%$ uncertainty level or support towards the data as

$$
\begin{array}{ll}
\text { Maximize } & R_{s}=\exp \left(-\lambda_{s} t\right) \\
\text { Minimize } & C_{s}=\sum_{j=1}^{7}\left\{a_{j} \log \left(\frac{1}{1-\exp \left(-\lambda_{j} t\right)}\right)+b_{j}\right\} \\
\text { s.t. } & (1-s) x_{k} \leq x_{k} \leq(1+s) x_{k}, \\
& x_{k}=\left[\lambda_{1}, \lambda_{2}, \ldots, \lambda_{7}, \tau_{1}, \tau_{2}, \ldots, \tau_{7}\right]^{T}, \\
& \lambda_{3}=\lambda_{4}=\lambda_{5}=\lambda_{6} ; \lambda_{7}=\lambda_{8} ; \lambda_{9}=\lambda_{10} \\
& \tau_{3}=\tau_{4}=\tau_{5}=\tau_{6} ; \tau_{7}=\tau_{8} ; \tau_{9}=\tau_{10}, \\
& t=10, \\
s & =0.15 \text { (considered uncertainty level), }
\end{array}
$$

where $\lambda_{s}$ is the system failure rate whose expression is given by

$$
\lambda_{s}=\lambda_{1}+\lambda_{2}+\lambda_{3}+\lambda_{4}+\lambda_{5}+\lambda_{6} \lambda_{7}\left(\tau_{6}+\tau_{7}\right) .
$$

The different values for the parameters $a_{j}(j=1,2, \ldots, 7)$ are $24,8,8.75,7.14,3.33,18$, and 18 , respectively, and for 
$b_{j}(j=1,2, \ldots, 7)$ are $120,80,70,50,30,50$, and 50, respectively, are chosen randomly.

\section{Computation Results}

This section turns to the description and analysis of the results obtained by the optimization tests.

6.1. Parameter Setting. In all algorithms, the values of the common parameters such as population size and total evaluation number are chosen to be the same. Population size and the maximum evaluation number are taken as $20 \times D$, where $D$ is dimension of the problem and 1500 , respectively, for the function. The method has been implemented in MATLAB (MathWorks); and in order to eliminate stochastic discrepancy, 30 independent runs have been made that involve 30 different initial trial solutions. The termination criterion has been set either limited to a maximum number of generations or to the order of relative error equal to $10^{-6}$, whichever is achieved first. The other randomly specified parameters of algorithms are given below.

6.1.1. GA Settings. In our experiment, real-coded genetic algorithm is utilized to find optimal values. Roulette wheel selection criterion is employed to choose better-fitted chromosomes. One-point crossover with the rate of 0.9 and random point mutation with the rate of 0.01 are used in the present analysis for the reproduction of new solution.

6.1.2. PSO Settings. Except common parameters (population number and maximum evaluation number), cognitive $\left(c_{1}\right)$ and social $\left(c_{2}\right)$ components are constants that can be used to change the weighting between personal and population experience, respectively. In our experiments, cognitive and the social components were both set to 1.49 . Inertia weight $(w)$, which determines how the previous velocity of the particle influences the velocity in the next iteration, was defined as the linearly decreases from initial weight $w_{1}=0.9$ to final weight $w_{2}=0.4$ with the relation $w=w_{2}+\left(\right.$ iter $_{\max }-$ iter) $\left(w_{1}-w_{2}\right) /$ iter $_{\max }$ where iter max $_{\text {ax }}$ is the maximum number of iteration, and iter is used iteration number [21].

6.2. Results and Discussions. The ideal values of the objective functions are given as $R_{s}^{l}=0.98046407, R_{s}^{u}=0.98554720$, $C_{s}^{l}=1039.731722$, and $C_{s}^{u}=1065.984110$. Based on these ideal values, a fuzzy region of satisfaction corresponding to the objective functions is to be constructed as follows:

$$
\mu_{C_{s}}(x)= \begin{cases}1 & C_{s}(x) \leqslant C_{s}^{l} \\ \frac{1065.984110-C_{s}(x)}{26.252388}, & C_{s}^{l} \leqslant C_{s}(x) \leq C_{s}^{u} \\ 0 & C_{s}(x) \geqslant C_{s}^{u},\end{cases}
$$

$$
\mu_{R_{s}}(x)= \begin{cases}1 & R_{s}(x) \geqslant R_{s}^{u} \\ \frac{R_{s}(x)-0.98046407}{0.00508313}, & R_{s}^{l} \leqslant R_{s}(x) \leqslant R_{s}^{u} \\ 0 & R_{s}(x) \leqslant R_{s}^{l} .\end{cases}
$$

Using these constructed membership functions and their weight vector as suggested by DM/system experts corresponding to the two objective functions, the equivalent crisp optimization problem is formulated as follows:

$$
\begin{array}{ll}
\text { Maximize: } & \left(1 \wedge \frac{\mu_{R_{s}}(x)}{w_{1}}\right) \wedge\left(1 \wedge \frac{\mu_{C_{s}}(x)}{w_{2}}\right) \\
\text { s.t. } \quad & (1-s) x_{k} \leq x_{k} \leq(1+s) x_{k}, \\
& w_{1}, w_{2} \in[0,1] .
\end{array}
$$

PSO has been used to solve (18) with the initial preference of the weight towards the objectives as $W 1=[1,1]$. Since in the first iteration DM does not want the preference towards the objectives; that is, they pay equal attention towards each objective. Hence, the weight vector is taken as 1 for each objective. Results corresponding to the first iteration are shown in Table 2, which is $\left(R_{s}, C_{s}\right)=(0.98359477,1049.92107)$ with membership value $\left(\mu_{R_{s}}, \mu_{C_{s}}\right)=(61.59 \%, 61.89 \%)$. Not satisfied with this outcome or willing to know other possible optimal solutions, keeping this result in view, let DM be decided to give more importance on reliability objective than cost objective and give a preference weight vector 1 corresponding to reliability and 0.5 to cost; that is, $W 2=[1,0.5]$. In other words, DM wants to pay two times more attention towards the reliability objective than cost objective. Outcome of this iteration is $\left(R_{s}, C_{s}\right)=(0.98451928,1055.59802)$ with membership value $\left(\mu_{R_{s}}, \mu_{C_{s}}\right)=(79.77 \%, 39.56 \%)$. Process is repeated till DM is fully satisfied. In this way, DM obtained the different possible solution for different satisfaction levels. Process is stopped after $V$ iteration (it may continue further till DM is satisfied). Outcome of iteration $V$ is $\left(R_{s}, C_{s}\right)=(0.98255695,1044.58702)$ with membership value $\left(\mu_{R_{s}}, \mu_{C_{s}}\right)=(41.17 \%, 81.50 \%)$. This result shows that $41.17 \%$ achievement for reliability and $81.50 \%$ for cost of respective fuzzy goals. A comparison of the results listed in the table with the results obtained by GA. It has been seen from the table that the results computed by PSO are better in terms of preferences given by the DM towards the objectives as compared to GA results. Thus, for different preferences suggested by DMs, optimum values of systems' reliability and cost are achieved. The optimum design parameters of design variables corresponding to optimum values are also summarized in Table 2. Based on these decision variables, the system analyst or decision makers may plan the schedule for proper maintenance in regular interval of time.

\section{Conclusion}

This paper reports the multiobjective optimization problem of the urea decomposition unit of a fertilizer plant under the fuzzy environment where we maximize the reliability 


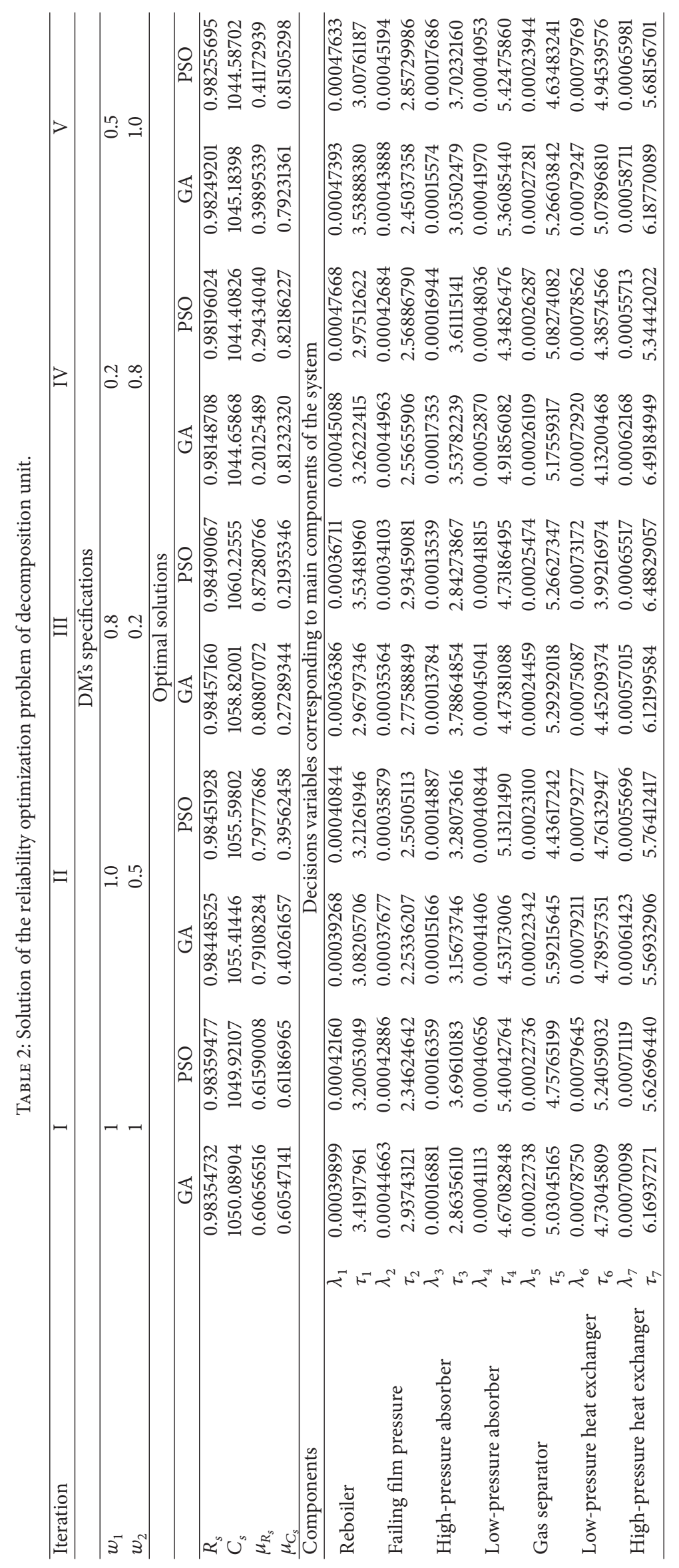


and minimize the cost of the system. A proposed approach suggests a way for achieving the optimum performance of the system with the help of experts/DMs elicitation. A mutual conflicting nature of the objectives is resolved with the help of fuzzy set theory after constructing the fuzzy region of satisfaction by taking linear membership functions. Since reliability decision is usually made in the earliest stage of system design and the information at this stage is incomplete and imprecise, it is necessary to rely on the experience of DMs and experts. Based on their preferences in the form of weights towards the objectives along with the achieved objective membership functions, FMOOP is reformulated as a single objective optimization problem and then solves with the PSO and GA iteratively. The one run of the approach has been shown here as we assume that in the problem DM is satisfied by the obtained results. Through this approach, a decision support system has been developed which helps the plant maintenance personnel in deciding his future strategy to gain optimum performance of the system. The decision variable corresponding to the main components of each subsystem/unit of a plant is reported which may be targeted so that optimum system performance could be achieved by using the discussed approach.

The present work done may be extended for a system in hybrid configuration, for example, series, parallel, seriesparallel, and so forth. The presented methodology will be further extended and improved using other optimization tools/algorithms and artificial neural network will be used to handle the complex nature of the systems. As a constant, failure rate model has been taken in the analysis, so authors are engaged in the approach for further analysis such as resource allocation, facility planning and management, inventory control, network analysis, and job shop scheduling for an arbitrary failure rate model instead of constant failure rate model.

\section{References}

[1] H. Garg, M. Rani, and S. P. Sharma, "Predicting uncertain behavior of press unit in a paper industry using artificial bee colony and fuzzy lambda-tau methodology," Applied Soft Computing, vol. 13, no. 4, pp. 1869-11881, 2013.

[2] H. Garg and S. P. Sharma, "Stochastic behavior analysis of industrial systems utilizing uncertain data," ISA Transactions, vol. 51, no. 6, pp. 752-762, 2012.

[3] C. Kai-Yuan, "Fuzzy reliability theories," Fuzzy Sets and Systems, vol. 40 , no. 3, pp. 510-511, 1991

[4] W. Karwowski and A. Mittal, Applications of Fuzzy Set Theory in Human Factors, Elsevier, Amsterdam, The Netherlands, 1986.

[5] A. K. Verma, A. Srividya, and R. S. P. Gaonkar, Fuzzy Reliability Engineering: Concepts and Applications, Narosa Publishing House, New Delhi, India, 2007.

[6] L. A. Zadeh, "Fuzzy sets," Information and Computation, vol. 8, pp. 338-353, 1965.

[7] R. E. Bellman and L. A. Zadeh, "Decision-making in a fuzzy environment," Management Science, vol. 17, pp. B141-B164, 1970.

[8] H. Garg and S. P. Sharma, "Multi-objective optimization of crystallization unit in a fertilizer plant using particle swarm optimization," International Journal of Applied Science and Engineering, vol. 9, no. 4, pp. 261-276, 2011.
[9] H. Garg and S. P. Sharma, "Multi-objective reliability-redundancy allocation problem using particle swarm optimization," Computers \& Industrial Engineering, vol. 64, no. 1, pp. 247-255, 2013.

[10] H. Garg, M. Rani, and S. P. Sharma, "Fuzzy RAM analysis of the screening unit in a paper industry by utilizing uncertain data," International Journal of Quality, Statistics and Reliability, vol. 2012, Article ID 203842, 14 pages, 2012.

[11] H. Z. Huang, "Fuzzy multi-objective optimization decisionmaking of reliability of series system," Microelectronics Reliability, vol. 37, no. 3, pp. 447-449, 1997.

[12] G. S. Mahapatra and T. K. Roy, "Fuzzy multi-objective mathematical programming on reliability optimization model," Applied Mathematics and Computation, vol. 174, no. 1, pp. 643659, 2006.

[13] K. S. Park, "Fuzzy apportionment of system reliability," IEEE Transactions on Reliability, vol. R-36, no. 1, pp. 129-132, 1987.

[14] M. Rani, S. P. Sharma, and H. Garg, "A novel approach for analyzing the behavior of repairable systems by utilizing uncertain data," International Journal of Performability Engineering, vol. 9, no. 2, pp. 201-210, 2013.

[15] M. Sakawa, "Multiobjective reliability and redundancy optimization of a series-parallel system by the Surrogate Worth Trade-off method," Microelectronics Reliability, vol. 17, no. 4, pp. 465-467, 1978.

[16] S. P. Sharma and H. Garg, "Behavioral analysis of a urea decomposition system in a fertilizer plant," International Journal of Industrial and System Engineering, vol. 8, no. 3, pp. 271-297, 2011.

[17] D. Kumar, Analysis and optimization of systems availability in sugar, paper and fertilizer industries [Ph.D. thesis], University of IIT Roorkee, Roorkee, India, 1991.

[18] K. K. Aggarwal and J. S. Gupta, "On minimizing the cost of reliable systems," IEEE Transactions on Reliability, vol. R-24, p. 205, 1975.

[19] R. Eberhart and J. Kennedy, "New optimizer using particle swarm theory," in Proceedings of the 6th International Symposium on Micro Machine and Human Science, pp. 39-43, October 1995.

[20] J. Kennedy and R. C. Eberhart, "Particle swarm optimization," in Proceedings of the IEEE International Conference on Neural Networks, vol. 4, pp. 1942-1948, Perth, Australian, December 1995.

[21] Y. Shi and R. C. Eberhart, "Parameter selection in particle swarm optimization," in Evolutionary Programming VII, pp. 591-600, Springer, New York, NY, USA, 1998. 


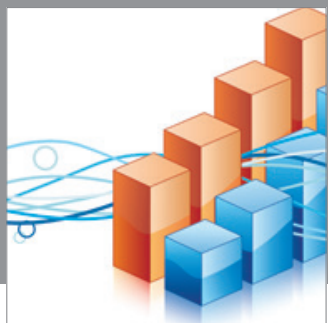

Advances in

Operations Research

mansans

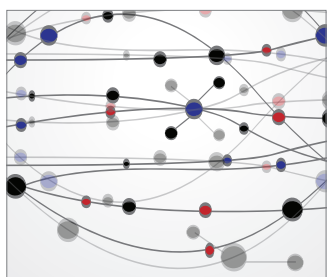

The Scientific World Journal
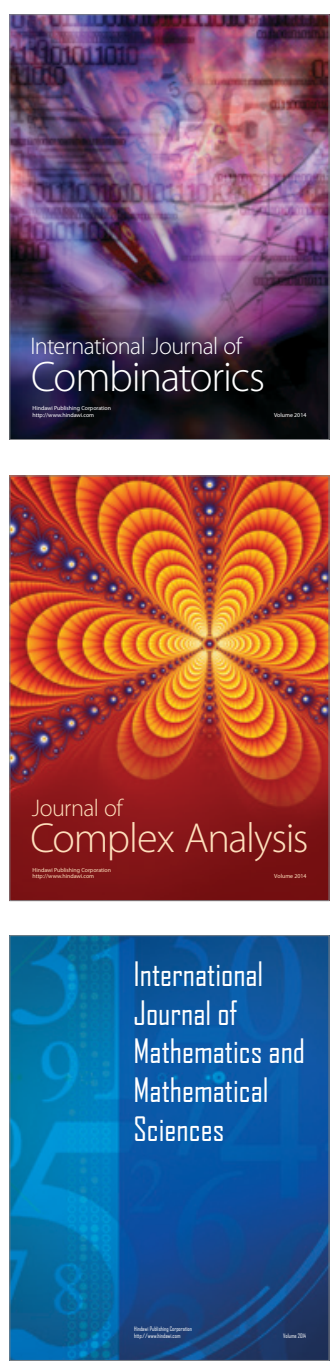
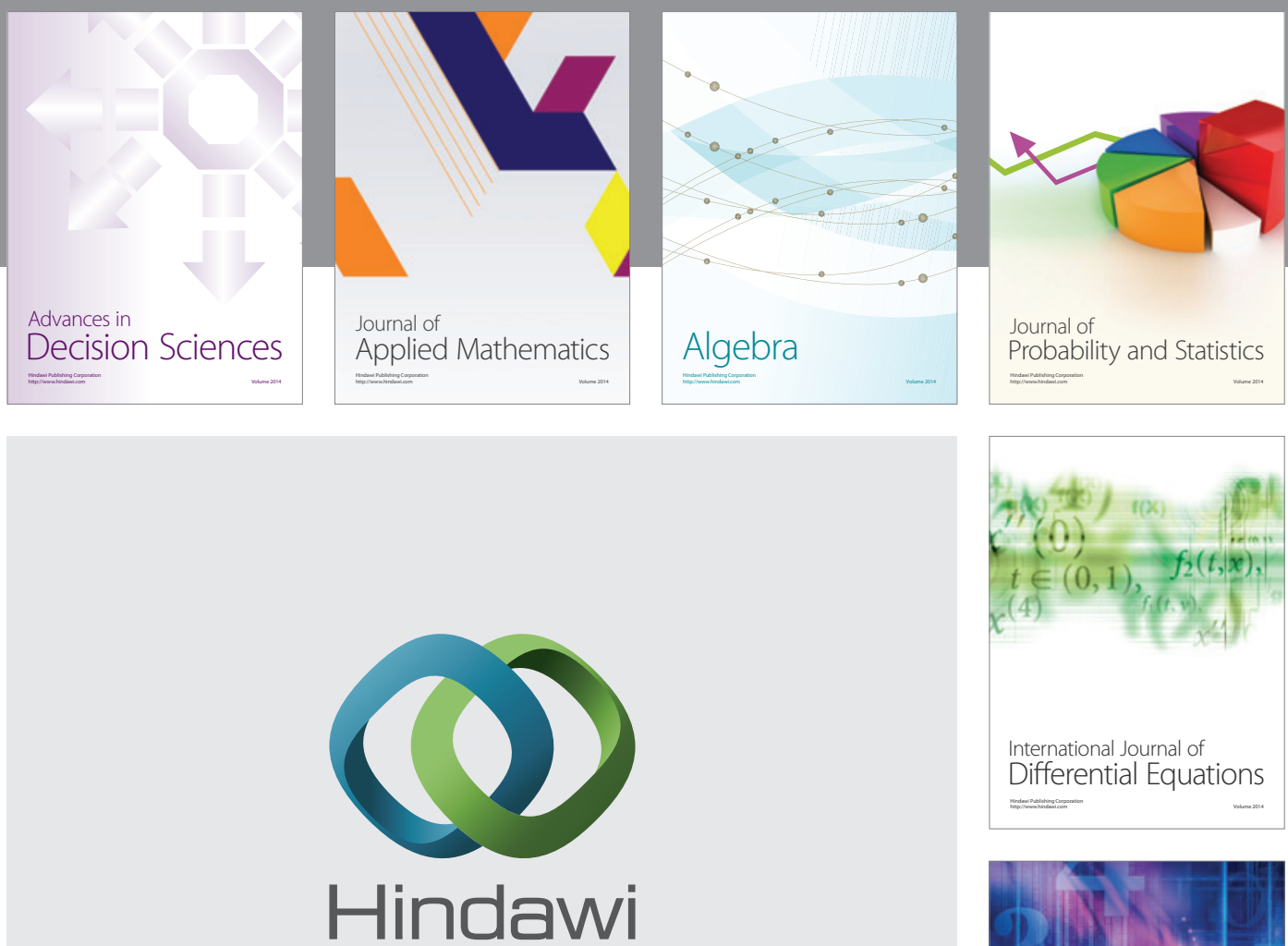

Submit your manuscripts at http://www.hindawi.com
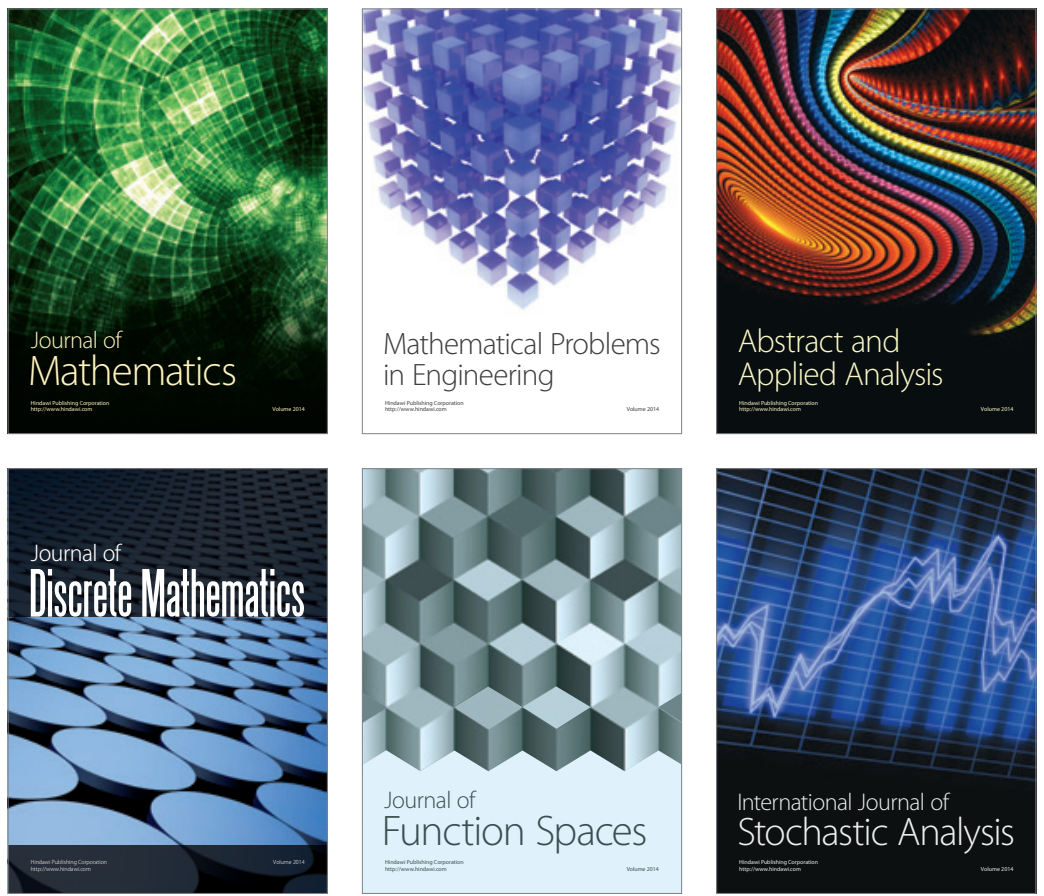

Journal of

Function Spaces

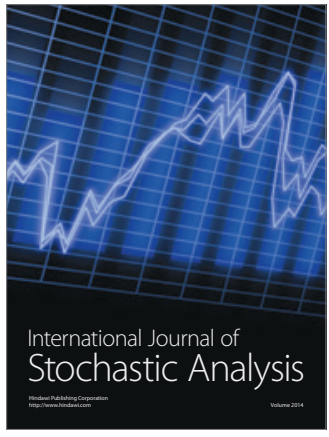

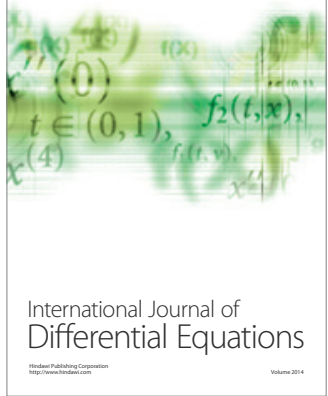
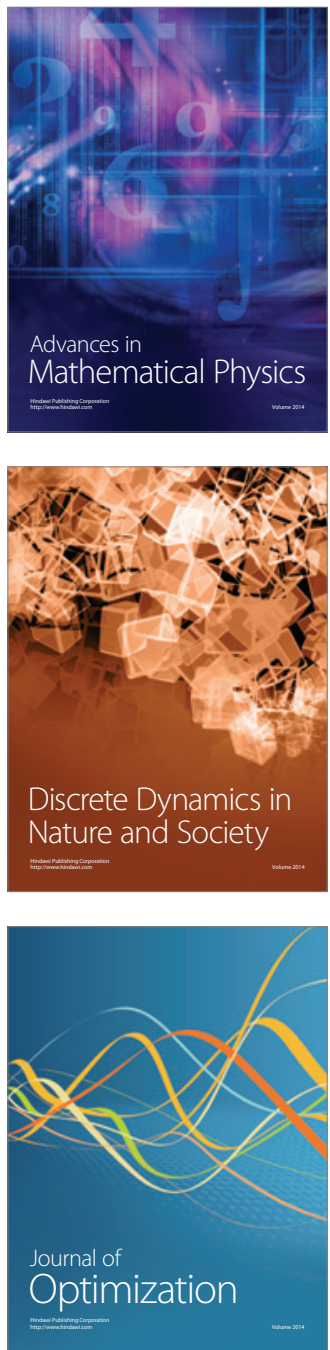